\title{
On Output Tracking Using Dynamic Output Feedback Discrete-Time Sliding Mode Controllers
}

\author{
Nai One Lai, Christopher Edwards and S.K. Spurgeon
}

\begin{abstract}
In this paper an output feedback based discretetime sliding mode control scheme is proposed. It incorporates a steady-state tracking requirement through the use of integral action. Previous work has shown that with an appropriate choice of sliding surface, discrete-time sliding mode control can be applied to non-minimum phase systems. The original scheme employed static output feedback and this imposed restrictions on the class of systems to which it was applicable - specifically a certain 'fictitious' sub-system was required to be output feedback stabilizable. The scheme proposed in this paper includes a compensator which broadens the class of systems for which the results are applicable. In the presence of bounded matched disturbances, ultimate boundedness results are obtained. It is also shown that in the presence of a class of sector bounded uncertainty, asymptotic stability can be achieved.
\end{abstract} LMIs

Index Terms-sliding modes, discrete-time, output feedback,

\section{INTRODUCTION}

$\mathbf{M}$ Any conventional (continuous-time) sliding mode control design schemes assume that all the states of the plant are directly accessible. In real systems this is not tenable and usually not all system states are fully available or measurable. One solution is to use an observer to reconstruct the system states [20], [23], [6]. Alternatively output feedback strategies can be employed in which the control law only requires knowledge of measured outputs [5], [8], [1]. There are, however, inherent system restrictions on using output feedback sliding mode control design methods: normally, the system must be relative degree one and minimum phase [7]. In some situations the relative degree condition can be relaxed by considering higher order sliding mode schemes. However the minimum phase restriction arises from the fact that the system zeros appear amongst the poles governing the sliding motion.

Compared with continuous time sliding mode strategies, the design problem in discrete-time has received much less coverage in the literature. With the exception of the early work in [19], most of the literature assumes all states are available [4], [9], [10], [11], [12], [22]. Schemes which have restricted themselves to output measurements alone have invariably utilized observers. Recent exceptions have been [14], [17] and the discrete-time versions of certain higher-order sliding mode control schemes in [2]. In particular, [14] considered an output tracking problem for an uncertain linear system using sliding mode ideas which requires output information only.

The authors are with the Control and Instrumentation Research Group, Department of Engineering, University of Leicester, University Road, Leicester, LE1 7RH, UK. Email: chris. edwards@le.ac.uk
It was shown in [14] that the relative degree and minimum phase requirements could be overcome by the use of a novel sliding surface. In order that a stable (ideal) discrete-time sliding motion exists, necessary and sufficient conditions were given in terms of the stabilizability, by static output feedback, of a fictitious system triple obtained from the real system. This fictitious system can easily be isolated once the real system is transformed into a special canonical form described in [14]. The stabilizability condition is a significant restriction on the class of systems to which the results are applicable, and of course for general multivariable systems this condition can, at best, only be tested numerically. In [14], a static output feedback structure was considered and so the fact that there is a limitation on the class of systems to which it is applicable is not surprising. This paper builds on this earlier work and proposes a specific compensator structure to circumvent this restriction. The resulting controller is applied to a High Incidence Research Model (HIRM) aircraft system as an example of a real engineering system.

\section{A Discrete-Time Sliding Mode Formulation}

Consider the square discrete-time system with matched uncertainties

$$
\begin{aligned}
x(k+1) & =G x(k)+H(u(k)+\xi(k)) \\
y(k) & =C x(k)
\end{aligned}
$$

where $x \in \mathbb{R}^{n}, u \in \mathbb{R}^{m}$ and $y \in \mathbb{R}^{p}$ with $m=p<n$. Assume that the input and output distribution matrices $H$ and $C$ are full rank. In addition, assume the triple $(G, H, C)$ is minimal. The matched uncertainties, represented by $\xi(k)$, are assumed to be unknown but bounded.

Consider the problem of determining an appropriate sliding surface $\mathcal{S}$ formed from a linear combination of the states, and a control law depending only on the measured outputs such that:

- for the nominal linear system when $\xi \equiv 0$ an ideal sliding motion is obtained in finite time i.e. $x(k) \in \mathcal{S}$ for all $k>k_{s}$;

- for uncertain systems the effect of the matched uncertainty $\xi$ is minimized and an appropriate bounded motion about $\mathcal{S}$ is maintained.

The discrete-time sliding mode situation is quite different from its continuous time counterpart: in continuous time a discontinuous control strategy can be employed to maintain ideal sliding in the presence of bounded matched uncertainty and in theory its effect is completely rejected; in discretetime, complete rejection of the uncertainty is not possible 
due to the sampled nature of the control signal. Furthermore, the inclusion of a switched term in the control law may be detrimental to the performance [21], [11], [12].

As in [21], the class of sliding surfaces will be restricted to those which can be expressed as

$$
\mathcal{S}=\left\{x \in \mathbb{R}^{n}: H^{\mathrm{T}} P x=0\right\}
$$

where $P \in \mathbb{R}^{n \times n}$ is a symmetric positive definite (s.p.d.) matrix. Associate with $P$ a candidate Lyapunov function $V(k)=x(k)^{\mathrm{T}} P x(k)$ and define a Lyapunov difference function by

$$
\Delta V(k)=V(k+1)-V(k)
$$

Consider initially a regulation problem where no uncertainty is present (i.e. $\xi(k) \equiv 0$ ). In the absence of uncertainty an ideal sliding motion can be attained on $\mathcal{S}$ whereby

$$
H^{\mathrm{T}} P x(k+1)=H^{\mathrm{T}} P G x(k)+H^{\mathrm{T}} P H u(k)=0
$$

It follows from (5) that the equivalent control action necessary to maintain an ideal sliding motion on $\mathcal{S}$ from (3) is given by

$$
u_{e q}(k)=-\left(H^{\mathrm{T}} P H\right)^{-1} H^{\mathrm{T}} P G x(k) .
$$

If $P$ is such that the closed-loop system, obtained from using the control law (6) in (1), satisfies $\Delta V(k)<0$ for all $k$, then from standard (discrete) Lyapunov theory the closed-loop system is asymptotically stable. It is clear that $\Delta V(k) \equiv-x^{\mathrm{T}}(k) Q x(k)$, where

$$
Q:=P-G_{c}^{\mathrm{T}} P G_{c}
$$

and the closed-loop system matrix

$$
G_{c}:=G-H\left(H^{\mathrm{T}} P H\right)^{-1} H^{\mathrm{T}} P G
$$

If $Q>0$, the closed-loop system is stable.

Remark 1: For the uncertain discrete-time system in (1), the control law (6), with $P$ chosen so that $Q$ from (7) is s.p.d, has the property that it:

a) induces an ideal sliding motion on $\mathcal{S}$ from (3) in finite time when $\xi(k) \equiv 0$ (this follows immediately from (5) and (6));

b) minimizes the effect of $\xi(k)$ on the closed loop dynamics in a min-max sense i.e. the control law minimizes over all possible state feedback control laws the effect of the worst case uncertainty $\xi(k)$ on the Lyapunov difference $\Delta V(k)$ (see [21]);

c) minimizes in a min-max sense the deviation from the ideal sliding surface $\mathcal{S}$ (see [11]).

If for a s.p.d. matrix $P$ satisfying $P-G_{c}^{\mathrm{T}} P G_{c}>0$ it is possible to solve

$$
H^{\mathrm{T}} P G=F C
$$

for some matrix $F \in \mathbb{R}^{m \times p}$ then provided $\operatorname{det} G \neq 0$, the controller from (6) can be realized through outputs alone as

$$
u(k)=-\left(F C G^{-1} H\right)^{-1} F y(k) .
$$

It is shown in [13] that two necessary conditions to solve the problem of synthesizing a s.p.d matrix $P$ satisfying (9) which ensures $Q$ from (7) is s.p.d, are
A1) the plant state transition matrix $G$ is nonsingular;

A2) the matrix $C G^{-1} H$ has rank $m$.

Based on assumptions $\mathrm{A} 1$ and $\mathrm{A} 2$, a change of coordinates can be introduced which facilitates insight into the class of systems for which this problem is solvable. Define a new matrix

$$
S:=C G^{-1} \text {. }
$$

This matrix will take the role of the output distribution matrix for a new, fictitious system $(G, H, S)$, which will be useful for the theoretical developments which follow. In order to facilitate the analysis, a change of coordinates will be introduced for the fictitious system $(G, H, S)$. From assumption $\mathrm{A} 2$ and the definition of $S$ in (11), $\operatorname{rank}(S H)=m$. As argued in [13], since $\operatorname{rank}(S H)=m$, there exists a change of coordinates such that $x \mapsto \bar{x}$ and $(G, H, S) \mapsto(\bar{G}, \bar{H}, \bar{S})$ where

$$
\bar{G}=\left[\begin{array}{ll}
G_{11} & G_{12} \\
G_{21} & G_{22}
\end{array}\right] ; \quad \bar{H}=\left[\begin{array}{c}
0 \\
H_{2}
\end{array}\right] ; \quad \bar{S}=\left[\begin{array}{cc}
0 & T
\end{array}\right]
$$

where $G_{11} \in \mathbb{R}^{(n-m) \times(n-m)}, H_{2} \in \mathbb{R}^{m \times m}$ and is nonsingular and $T \in \mathbb{R}^{m \times m}$ is orthogonal. As argued in [13], necessary and sufficient conditions to solve the problem of synthesizing a s.p.d matrix $P$ satisfying (9) which ensures $Q$ from (7) is s.p.d, are that A1 and A2 hold, together with a third requirement:

A3) the matrix sub-block $G_{11}$ from (12) is stable.

The coordinate system associated with (12) will be used as a basis for the results which follow. ${ }^{1}$

Remark 2: Assumption A1 appears in all the discrete-time output min-max literature: see for example [18]. However A1 means the approach in this paper is not applicable to discretetime systems which contain pure time delays. Condition A2 is a necessary condition to find a s.p.d. matrix $P$ and an $F \in \mathbb{R}^{m \times p}$ to solve (9). This can be easily verified: assuming $\operatorname{det}(G) \neq 0$, if (9) is satisfied then $H^{\mathrm{T}} P H=F C G^{-1} H$ and hence $\operatorname{rank}\left(F C G^{-1} H\right)=m$. This implies $C G^{-1} H$ must be rank $m$. The second condition is equivalent to the triple $(G, H, C)$ not having any invariant zeros at the origin since $C G^{-1} H=\mathcal{G}(0)$ where $\mathcal{G}(z):=C(z I-G)^{-1} H$ and so $\operatorname{rank}\left(C G^{-1} H\right)=m$ implies $z=0$ is not an invariant zero. Condition A3 is limiting and will be obviated in this paper by the introduction of a compensator.

Remark 3: It can be shown [13] that A3 is equivalent to the triple $(G, H, S)$ being minimum phase since the eigenvalues of $G_{11}$ represent the invariant zeros of $(G, H, S)$. Note: this is quite different to the continuous-time min-max case where the system representation from the inputs to the true outputs must be minimum phase. As argued in [13], [14] it is quite possible for $(G, H, C)$ to be non-minimum phase whilst satisfying A3.

This paper will consider the situation where a tracking requirement is required and will remove $\mathrm{A} 3$ by the use of a suitable dynamic compensator.

\section{MAIN RESUlts}

Assume throughout the rest of the paper that A1 and A2 from $\S I I$ hold. It follows from the canonical form (12) that the

\footnotetext{
${ }^{1}$ In fact [13] considers the more general situation where $p \geq m$ and a slightly more elaborate version of $\mathrm{A} 3$ is proved.
} 
true output distribution matrix

$$
\bar{C}=\bar{S} \bar{G}=\left[\begin{array}{ll}
T G_{21} & T G_{22}
\end{array}\right]
$$

To incorporate a tracking element, integral action will also be included. The difference equation

$$
x_{r}(k+1)=x_{r}(k)+\tau(r(k)-y(k))
$$

will be added where $\tau$ represents the sample interval ${ }^{2}$. The quantity $r(k)$ represents the signal to be tracked by the output. Furthermore assume $r(k)=r_{s}=$ const for $k>k_{0}$.

Partition the state vector $\bar{x}$ conformably as $\operatorname{col}\left(x_{1}, x_{2}\right)$ where $x_{1} \in \mathbb{R}^{(n-m)}$. Also introduce additional states $x_{c} \in$ $\mathbb{R}^{(n-m)}$, which under certain circumstances represent an estimate of the states $x_{1}$.

The intention is to induce an ideal sliding motion on

$$
\mathcal{S}=\left\{\left(x_{1}, x_{c}, x_{r}, x_{2}\right): K_{1} x_{c}+K_{r} x_{r}+x_{2}+S_{r} r_{s}=0\right\},
$$

where $K_{1} \in \mathbb{R}^{m \times(n-m)}$ and $K_{r} \in \mathbb{R}^{m \times m}$ together with $S_{r} \in \mathbb{R}^{m \times m}$ represent design freedom. Let the compensator take the form

$$
x_{c}(k+1)=G_{11} x_{c}(k)+G_{12} x_{2}(k)+L(y(k)-\hat{y}(k))
$$

where

$$
\hat{y}(k):=T G_{21} x_{c}(k)+T G_{22} x_{2}(k)
$$

and $L \in \mathbb{R}^{(n-m) \times m}$ is a design variable. During an ideal sliding motion, from (15),

$$
x_{2}(k)=-K_{1} x_{c}(k)-K_{r} x_{r}(k)-S_{r} r(k)
$$

and so after some algebraic manipulation it can be shown that

$$
x_{c}(k+1)=\Phi x_{c}(k)+\Gamma_{1} y(k)+\Gamma_{2} x_{r}(k)+\Gamma_{3} r(k)
$$

where

$$
\Phi=G_{11}-L T G_{21}-G_{12} K_{1}+L T G_{22} K_{1}
$$

and

$$
\begin{aligned}
& \Gamma_{1}=L, \\
& \Gamma_{2}=-G_{12} K_{r}+L T G_{22} K_{r} \\
& \Gamma_{3}=-G_{12} S_{r}+L T G_{22} S_{r} .
\end{aligned}
$$

It is assumed that as part of the design process, $L$ is chosen to guarantee that $\operatorname{det} \Phi \neq 0$. Augment the system in (1), in the canonical form of (12), with the integral and compensator states from (14) and (18) to obtain:

$$
x_{a}(k+1)=G_{a} x_{a}(k)+H_{a}(u(k)+\xi(k))+H_{r} r(k),
$$

where $x_{a}=\operatorname{col}\left(x_{1}, x_{c}, x_{r}, x_{2}\right)$. (At first sight this represents a non-intuitive arrangement of the states but it leads to a simplification in the presentation.)

\footnotetext{
${ }^{2}$ If (1)-(2) is a genuinely discrete system and does not arise from sampling a continuous-time system, then (14) can be replaced by $x_{r}(k+1)=x_{r}(k)+$ $r(k)-C_{p} x_{p}(k)$ and all the results which will subsequently be proved are still true when this equation is used in place of (14).
}

The avialable outputs associated with this system are given by $y_{a}=\operatorname{col}\left(x_{c}, x_{r}, y\right)$. It is easily verified that

$$
G_{a}=\left[\begin{array}{cccc}
G_{11} & 0 & 0 & G_{12} \\
\Gamma_{1} T G_{21} & \Phi & \Gamma_{2} & \Gamma_{1} T G_{22} \\
-\tau T G_{21} & 0 & I_{m} & -\tau T G_{22} \\
G_{21} & 0 & 0 & G_{22}
\end{array}\right] ; H_{a}=\left[\begin{array}{c}
0 \\
0 \\
0 \\
H_{2}
\end{array}\right]
$$

and the output distribution matrix

$$
C_{a}=\left[\begin{array}{cccc}
0 & I_{n-m} & 0 & 0 \\
0 & 0 & I_{m} & 0 \\
T G_{21} & 0 & 0 & T G_{22}
\end{array}\right],
$$

where $y_{a}:=C_{a} x_{a}$. Modify the control law in (10) to include the reference signal so that

$$
u(k)=-\left(F_{a} C_{a} G_{a}^{-1} H_{a}\right)^{-1} F_{a} C_{a} x_{a}(k)+F_{r} r(k),
$$

where now both $F_{a}$ and $F_{r} \in \mathbb{R}^{m \times m}$ are to be determined (in terms of $L, K_{1}, K_{r}$ and $S_{r}$ ). The objective is to select $F_{a}$ and a matrix $F_{2} \in \mathbb{R}^{m \times m}$ so that the surface

$$
\mathcal{S}_{a}=\left\{x_{a}: F_{a} C_{a} G_{a}^{-1} x_{a}+F_{2} S_{r} r_{s}=0\right\}
$$

is identical to the surface $\mathcal{S}$ in (15), and then to select $K_{1}, K_{r}$ and $L$ to ensure a stable ideal sliding motion when confined to $\mathcal{S}$.

Providing the design matrix $F_{a}$ is chosen to ensure the eigenvalues of

$$
G_{c}=G_{a}-H_{a}\left(F_{a} C_{a} G_{a}^{-1} H_{a}\right) F_{a} C_{a}
$$

are inside the unit disk, $\left(I-G_{c}\right)$ is invertible. Define $x_{s}=$ $\left(I-G_{c}\right)^{-1}\left(H_{r}+H_{a} F_{r}\right) r_{s}$ then using (26) and defining

$$
e(k)=x_{a}(k)-x_{s}
$$

it follows from simple algebraic manipulation that

$$
e(k+1)=G_{c} e(k)+H_{a} \xi(k)
$$

In the absence of uncertainty $e(k) \rightarrow 0$ as $k \rightarrow \infty$, and since steady state is achieved, it follows from (14) that $y(k)=r_{s}$ and so tracking is achieved. Furthermore it can be shown that

$$
\begin{aligned}
F_{a} C_{a} G_{a}^{-1} x_{s} & =F_{a} C_{a} G_{a}^{-1}\left(I-G_{c}\right)^{-1}\left(H_{r}+H_{a} F_{r}\right) r_{s} \\
& \equiv F_{a} C_{a} G_{a}^{-1}\left(H_{r}+H_{a} F_{r}\right) r_{s}
\end{aligned}
$$

and consequently if $F_{r}$ is chosen as

$$
F_{r}:=-\left(F_{a} C_{a} G_{a}^{-1} H_{a}\right)^{-1}\left(F_{a} C_{a} G_{a}^{-1} H_{r}+F_{2} S_{r}\right)
$$

then $F_{a} C_{a} G_{a}^{-1} x_{s}+F_{2} S_{r} r_{s}=0$ and so $x_{s} \in \mathcal{S}$. From (30), and following similar arguments to those presented in $\S I I$ concerning (7) and (9), the problem is therefore to find an $F_{a}$ and a s.p.d. matrix $P_{a} \in \mathbb{R}^{2 n \times 2 n}$ such that

$$
F_{a} C_{a}=H_{a}^{\mathrm{T}} P_{a} G_{a}
$$

and

$$
G_{c}^{\mathrm{T}} P_{a} G_{c}-P_{a}<0
$$

Proposition 1: Assuming conditions A1 and A2 are satisfied, then there exist matrices $F_{a}$ and $P_{a}$ such that (32)-(33) hold. 
Proof If A1 and A2 are satisfied then all the development in $\S I I I$ is valid. Define for the augmented system (30) and the output distribution matrix in (25) a fictitious output distribution matrix $S_{a}:=C_{a} G_{a}^{-1}$. After some algebra it can be shown that

$$
\begin{aligned}
S_{a} & =\left[\begin{array}{c|ccc}
0 & \Phi^{-1} & -\Phi^{-1} \Gamma_{2} & -\Phi^{-1} \Gamma_{1} T-\tau \Phi^{-1} \Gamma_{2} T \\
0 & 0 & I_{m} & \tau T \\
0 & 0 & 0 & T
\end{array}\right] \\
& =:\left[\begin{array}{ll}
0 & T_{a}
\end{array}\right],
\end{aligned}
$$

where $T_{a} \in \mathbb{R}^{(n+m) \times(n+m)}$ and $\operatorname{det} T_{a} \neq 0$. Define a matrix

$$
F_{a}:=F_{2}\left[\begin{array}{lll}
K_{1} \Phi & K_{1} \Gamma_{2}+K_{r} & K_{1} \Gamma_{1}-K_{r} \tau+T^{\mathrm{T}}
\end{array}\right],
$$

where $F_{2} \in \mathbb{R}^{m \times m}$ and is nonsingular. The matrix $F_{2}$ has no effect on the dynamics of the ideal sliding motion but is required to solve the constraint (32). After a little algebra it can be shown that

$$
F_{a} S_{a}=F_{a} C_{a} G_{a}^{-1}=F_{2}\left[\begin{array}{llll}
0 & K_{1} & K_{r} & I_{m}
\end{array}\right] .
$$

To facilitate choosing the parameters $L, K_{1}$ and $K_{r}$, change coordinates according to the transformation $x_{a} \mapsto \tilde{T} x_{a}=: \tilde{x}$ where

$$
\tilde{T}:=\left[\begin{array}{cccc}
I_{n-m} & -I_{n-m} & 0 & 0 \\
0 & I_{n-m} & 0 & 0 \\
0 & 0 & I_{m} & 0 \\
0 & K_{1} & K_{r} & I_{m}
\end{array}\right] .
$$

This effectively forces the last $m$ states of the new coordinates to represent what, in continuous-time sliding mode control, is called the 'switching function' $\sigma=K_{r} x_{r}+K_{1} x_{c}+x_{2}$ associated with the surface $\mathcal{S}$ in (15). It follows that the matrices $\tilde{G}=\tilde{T} G_{a} \tilde{T}^{-1}, \tilde{H}=\tilde{T} H_{a}, \tilde{H}_{r}=\tilde{T} H_{r}, \tilde{C}=C_{a} \tilde{T}^{-1}$ and $\tilde{S}=S_{a} \tilde{T}^{-1}$. After some straightforward algebra

$$
\tilde{H}=\left[\begin{array}{c}
0 \\
0 \\
0 \\
H_{2}
\end{array}\right] \quad \text { and } \quad \tilde{H}_{r}=\left[\begin{array}{c}
-\Gamma_{3} \\
\Gamma_{3} \\
\tau I_{m} \\
K_{1} \Gamma_{3}+\tau K_{r}
\end{array}\right] .
$$

From equation (36) it follows that

$$
F_{a} \tilde{S}=\left[\begin{array}{llll}
0 & 0 & 0 & F_{2}
\end{array}\right] .
$$

Some algebra reveals the closed-loop system matrix

$$
\tilde{G}_{c}=\tilde{G}-\tilde{H}\left(F_{a} \tilde{S} \tilde{H}\right)^{-1} F_{a} \tilde{C}=:\left[\begin{array}{cc}
\tilde{G}_{11} & \tilde{G}_{12} \\
0 & 0
\end{array}\right],
$$

where

$$
\tilde{G}_{11}=\left[\begin{array}{c|c}
G_{11}-L T G_{21} & 0 \\
\hline L T G_{21} & \tilde{G}_{m} \\
-\tau T G_{21} &
\end{array}\right]
$$

and

$$
\tilde{G}_{m}:=\left[\begin{array}{cc}
G_{11}-G_{12} K_{1} & -G_{12} K_{r} \\
-\tau T G_{21}+\tau T G_{22} K_{1} & I_{m}+\tau T G_{22} K_{r}
\end{array}\right]
$$

This is most easily seen from the definition of $\tilde{S}=\tilde{C} \tilde{G}^{-1}$ and the fact that $\tilde{G}_{c}=\left(I-\tilde{H}\left(F_{a} \tilde{S} \tilde{H}\right)^{-1} F_{a} \tilde{S}\right) \tilde{G}$. From (39) and (38) it can be easily shown that

$$
\left(I-\tilde{H}\left(F_{a} \tilde{S} \tilde{H}\right)^{-1} F_{a} \tilde{S}\right)=\operatorname{diag}\left(I_{n-m}, I_{n-m}, I_{m}, 0_{m \times m}\right)
$$

and hence the structure in (40) follows immediately. It is clear from (40) and (41) that

$$
\sigma\left(\tilde{G}_{c}\right)=\{0\}^{m} \cup \sigma\left(G_{11}-L T G_{21}\right) \cup \sigma\left(\tilde{G}_{m}\right),
$$

where

$$
\tilde{G}_{m}=\underbrace{\left[\begin{array}{cc}
G_{11} & 0 \\
-\tau T G_{21} & I_{m}
\end{array}\right]}_{G_{11}^{a}}-\underbrace{\left[\begin{array}{c}
G_{12} \\
-\tau T G_{22}
\end{array}\right]}_{G_{12}^{a}}\left[\begin{array}{ll}
K_{1} & K_{r}
\end{array}\right]
$$

and $\sigma(\cdot)$ denotes the spectrum of a matrix. Since the matrix pair $\left(G_{11}, G_{21}\right)$ is observable (see for example [13]) and $T$ is nonsingular, the pair $\left(G_{11}, T G_{21}\right)$ is observable. Consequently $L$ can be chosen to make $\left(G_{11}-L T G_{21}\right)$ stable. Likewise it can be shown that provided $(G, H, C)$ does not have invariant zeroes at unity, the pair $\left(G_{11}^{a}, G_{12}^{a}\right)$ is controllable and hence the choice of $K_{1}$ and $K_{r}$ constitutes a state-feedback problem. Consequently $K_{1}, K_{r}$ and $L$ can be chosen to make $\tilde{G}_{11}$ from (41) stable. In the new set of coordinates $\tilde{x}$, let the Lyapunov matrix be represented by $\tilde{P}$. Using the definition of $\tilde{S}$, equation (32) becomes

$$
\tilde{H}^{\mathrm{T}} \tilde{P}=F_{a} \tilde{C} \tilde{G}^{-1}=F_{a} \tilde{S}
$$

In order to show that $\tilde{P}$ is a Lyapunov matrix for $\tilde{G}_{c}$ it must be established that

$$
\tilde{Q}:=\tilde{P}-\tilde{G}_{c}^{\mathrm{T}} \tilde{P} \tilde{G}_{c}>0
$$

It can be seen from the structures of $\tilde{H}$ and $F_{a} \tilde{S}$ in (38) and (39) and from the fact that $\operatorname{det} H_{2} \neq 0$ that in order to satisfy (44), $\tilde{P}$ must have a block diagonal structure:

$$
\tilde{P}=\left[\begin{array}{cc}
\tilde{P}_{1} & 0 \\
0 & \tilde{P}_{2}
\end{array}\right],
$$

where $\tilde{P}_{1} \in \mathbb{R}^{(2 n-m) \times(2 n-m)}, \tilde{P}_{2} \in \mathbb{R}^{m \times m}$ and

$$
F_{2}=H_{2}^{\mathrm{T}} \tilde{P}_{2} .
$$

In terms of the partition in (40), (45) can be written as

$$
\tilde{Q}=\left[\begin{array}{cc}
\tilde{P}_{1}-\tilde{G}_{11}^{\mathrm{T}} \tilde{P}_{1} \tilde{G}_{11} & -\tilde{G}_{11}^{\mathrm{T}} \tilde{P}_{1} \tilde{G}_{12} \\
-\tilde{G}_{12}^{\mathrm{T}} \tilde{P}_{1} \tilde{G}_{11} & \tilde{P}_{2}-\tilde{G}_{12}^{\mathrm{T}} \tilde{P}_{1} \tilde{G}_{12}
\end{array}\right] .
$$

Let $\tilde{P}_{1}>0$ be a solution to

$$
\tilde{P}_{1}-\tilde{G}_{11}^{\mathrm{T}} \tilde{P}_{1} \tilde{G}_{11}>0 .
$$

Such a solution $\tilde{P}_{1}$ is guaranteed to exist since $\tilde{G}_{11}$ is stable. Then from the Schur complement, inequality (48) is satisfied if and only if

$$
\begin{aligned}
\tilde{P}_{2}> & \tilde{G}_{12}^{\mathrm{T}} \tilde{P}_{1} \tilde{G}_{11}\left(\tilde{P}_{1}-\tilde{G}_{11}^{\mathrm{T}} \tilde{P}_{1} \tilde{G}_{11}\right)^{-1}\left(\tilde{G}_{11}^{\mathrm{T}} \tilde{P}_{1} \tilde{G}_{12}\right) \\
& +\tilde{G}_{12}^{\mathrm{T}} \tilde{P}_{1} \tilde{G}_{12}
\end{aligned}
$$

Any pair $\left(\tilde{P}_{1}, \tilde{P}_{2}\right)$ satisfying (49) and (50) ensures $\tilde{P}$ from (46) satisfies (44) and (45). Therefore $F_{a}$ as defined in (35) and $P_{a}=\tilde{T}^{\mathrm{T}} \tilde{P} \tilde{T}$ where $\tilde{T}$ is given in (37) constitutes a solution to (32)-(33) and the proposition is proved.

Corollary 1: The sliding surface $\mathcal{S}_{a}$ given in (27) is identical to $\mathcal{S}$ given in (15).

Proof From the choice of $F_{r}$ in (31) and $F_{a} C_{a} G_{a}^{-1}$ in (36), the equivalence of $\mathcal{S}_{a}$ with $\mathcal{S}$ from (15) is clear since $F_{2}$ is nonsingular. 
Remark 4: It is easy to see from that the control law is independent of the choice of matrix $F_{2}$.

\section{Robustness}

This subsection considers the robustness properties of the controller developed in $\S$ III. Suppose the matched uncertainty $\xi(k)$ in (1), in the coordinates associated with (12), satisfies

$$
\|\xi(k)\|<\rho_{1}\|\bar{x}(k)\|+\rho_{0},
$$

where $\rho_{1}$ and $\rho_{0}$ are positive constants. Let

$$
N:=\left[\begin{array}{cccc}
I_{n-m} & 0 & 0 & 0 \\
0 & 0 & 0 & I_{m}
\end{array}\right] .
$$

In this section, assume initially that $r(k) \equiv 0 \forall k$. Then $\bar{x}=N x_{a}=N e$ where $e$ is defined in (29) since $x_{s}=0$ because $r_{s}=0$ by hypothesis. Consequently inequality (51) can be written as

$$
\|\xi(k)\|<\rho_{1}\|N e(k)\|+\rho_{0} .
$$

The design freedom associated with the Lyapunov matrix has been shown to be represented by the pair of s.p.d. matrices $\tilde{P}_{1}$ and $\tilde{P}_{2}$. Although the pair $\left(\tilde{P}_{1}, \tilde{P}_{2}\right)$ must satisfy the matrix inequalities (49) and (50), there is some inherent design freedom. The selection of these matrices has no effect on the compensator dynamics (18) or indeed the control law. Assume the relevant design parameters have been selected to ensure $\tilde{G}_{c}$ (and in particular $\tilde{G}_{11}$ from (40)) is stable. Define

$\mathcal{L}\left(\tilde{P}_{1}, \tilde{P}_{2}, \mu\right):=-e^{\mathrm{T}} \tilde{T}^{\mathrm{T}} \tilde{Q} \tilde{T} e+\xi^{\mathrm{T}} \tilde{H}^{\mathrm{T}} \tilde{P} \tilde{H} \xi+\left(\mu e^{\mathrm{T}} N^{\mathrm{T}} N e-\xi^{\mathrm{T}} \xi\right)$

where $\tilde{Q}$ is defined in (48), $\tilde{T}$ is given in (37), $\tilde{P}$ is defined in (46) and the scalar $\mu>0$.

Proposition 2: Suppose $\tilde{P}_{1}, \tilde{P}_{2}$ and $\mu$ are chosen so that $\mathcal{L}\left(\tilde{P}_{1}, \tilde{P}_{2}, \mu\right)<0$ and $\rho_{1}<\sqrt{\mu}$, then in the absence of external disturbances (i.e. when $\rho_{0}=0$ ), asymptotic stability of the closed-loop system (30) is guaranteed.

Proof Let

$$
V(k):=e^{\mathrm{T}}(k) P_{a} e(k),
$$

where $P_{a}:=\tilde{T}^{\mathrm{T}} \tilde{P} \tilde{T}$. Then from (30) it follows that

$$
\Delta V(k)=-e^{\mathrm{T}}(k) \tilde{T}^{\mathrm{T}} \tilde{Q} \tilde{T} e(k)+\xi^{\mathrm{T}}(k) \underbrace{\tilde{H}^{\mathrm{T}} \tilde{P} \tilde{H}}_{=H_{a}^{\mathrm{T}} P_{a} H_{a}} \xi(k),
$$

Since by hypothesis $\rho_{1}<\sqrt{\mu}$ and $\rho_{0}=0$, it follows from (53) that $\|\xi\|<\sqrt{\mu}\|N e\|$. Consequently $\mu e^{\mathrm{T}} N^{\mathrm{T}} N e-\xi^{\mathrm{T}} \xi>0$, which in conjunction with (54), implies $\Delta V(k)<0$. It then follows from standard Lyapunov arguments that $e(k) \rightarrow 0$ as $k \rightarrow \infty$ and asymptotic stability is proved.

Corollary 2: The states $e(k)$ are forced to evolve in such a way that the deviation from $\mathcal{S}_{a}$ tends to zero with respect to time.

Proof It follows from (30) that

$$
F_{a} C_{a} G_{a}^{-1} e(k+1)=H_{a}^{T} P_{a} H_{a} \xi(k)
$$

for $k=1,2, \ldots$ and thus $\left\|H_{a}^{T} P_{a} H_{a} \xi(k)\right\|$ represents the deviation from the ideal sliding surface

$$
\begin{aligned}
\mathcal{S}_{a} & =\left\{x_{a} \mid F C_{a} G_{a}^{-1} x_{a}+F_{2} S_{r} r_{s}=0\right\} \\
& \equiv\left\{e \mid F C_{a} G_{a}^{-1} e=0\right\}
\end{aligned}
$$

As argued above $\|\xi(k)\| \leq \rho_{1}\|N e(k)\|$ for all $k$ and thus $\left\|H_{a}^{T} P_{a} H_{a} \xi(k)\right\| \leq \rho_{1}\left\|H_{a}^{T} P_{a} H_{a}\right\|\|e(k)\| \rightarrow 0$ as $k \rightarrow \infty$ as claimed, since $\|e(k)\| \rightarrow 0$ from Proposition 2.

The condition $\mathcal{L}\left(\tilde{P}_{1}, \tilde{P}_{2}, \mu\right)<0$ is guaranteed if

$$
\begin{aligned}
\mu N^{\mathrm{T}} N & <\tilde{T}^{\mathrm{T}} \tilde{Q} \tilde{T}, \\
\tilde{H}^{\mathrm{T}} \tilde{P} \tilde{H} & <I_{m}
\end{aligned}
$$

are satisfied subject to $\tilde{P}>0$. A logical way to proceed is to choose $\tilde{P}_{1}, \tilde{P}_{2}$ satisfying (58)-(59) to maximize $\mu$. This represents a convex optimization problem with decision variables $\tilde{P}_{1}, \tilde{P}_{2}$ and $\mu$. Linear Matrix Inequality (LMI) methods [3] can be used to obtain the optimal values of the decision matrices as a generalized eigenvalue problem.

Finally if $\rho_{0} \neq 0$ and/or $r_{s} \neq 0$ then (51) becomes

$$
\|\xi(k)\|<\rho_{1}\|N e(k)\|+\underbrace{\left(\rho_{0}+\rho_{1}\left\|N x_{s}\right\|\right)}_{\bar{\rho}_{0}}
$$

rather than (53). Now quadratic stability is lost, but if $\rho_{1}<\sqrt{\mu}$, ultimate boundedness can still be guaranteed using arguments similar to those in Proposition 2.

\section{EXAMPLE}

In this section the longitudinal dynamics of the High Incidence Research Model (HIRM) aircraft will be considered [16]. A linearization of the nonlinear simulation [16] about Mach number 0.3 and an altitude of $5000 \mathrm{ft}$ has been used as the basis of the design. A discretized representation based on a sample interval of 0.025 secs is

$G=\left[\begin{array}{ccc}0.9862 & 0.0243 & 0 \\ -0.0264 & 0.9894 & 0 \\ -0.0003 & 0.0249 & 1.0000\end{array}\right] ; H=\left[\begin{array}{c}-0.0038 \\ -0.0810 \\ -0.0010\end{array}\right]$;

where $C=\left[\begin{array}{lll}0 & 0 & 1\end{array}\right]$ the states of the model are angle of attack (rad), pitch rate ( $\mathrm{rad} / \mathrm{s})$ and pitch angle (rad). The inputs and outputs are symmetrical tail plane deflection and pitch respectively. In the coordinates of (12) it can be shown that

$$
\left[\begin{array}{ll}
G_{11} & G_{12} \\
G_{21} & G_{22}
\end{array}\right]=\left[\begin{array}{cc|c}
0.9874 & -0.1166 & 9.1989 \\
-0.0003 & 2.9806 & -157.0127 \\
\hline-0.0003 & 0.0251 & -0.9925
\end{array}\right]
$$

and $T=1$. Choosing

$$
L=\left[\begin{array}{c}
-4.5025 \\
80.2795
\end{array}\right]
$$

and

$$
\left[\begin{array}{ll}
K_{1} & K_{r}
\end{array}\right]=\left[\begin{array}{ll}
0.4004 & 0.0090 \mid 0.6418
\end{array}\right]
$$

means $\sigma\left(G_{11}-L T G_{21}\right)=\{0.97,0.98\}$ and $\sigma\left(\tilde{G}_{m}\right)=$ $\{0.80,0.90,0.98\}$. In the following design $S_{r}=0.005$ has been selected. From equations (19)-(22) it follows that

$$
\Phi=\left[\begin{array}{rr}
-0.9082 & -0.0460 \\
30.9949 & 1.6593
\end{array}\right]
$$

and

$$
\left[\begin{array}{lll}
\Gamma_{1} & \Gamma_{2} & \Gamma_{3}
\end{array}\right]=\left[\begin{array}{c|c|c}
-4.5025 & -3.0357 & -0.0237 \\
80.2795 & 49.6316 & 0.3867
\end{array}\right],
$$


It can be verified that $\operatorname{det}(\Phi)=-0.0798$ and so $\Phi$ is invertible as required by the theory. From (35) it can be shown

$$
F_{a}=F_{2}\left[\begin{array}{llll}
-0.1277 & -0.0851 & -0.0035 & -0.0974
\end{array}\right]
$$

where $F_{2}$ is a non-zero scalar. From (26)

$$
\begin{aligned}
u(k)=[ & {\left[\begin{array}{llll}
-124.864 & -83.191 & -3.445 & -95.211
\end{array}\right] y_{a}(k) } \\
& +9.7553 r(k) .
\end{aligned}
$$

The LMI optimization gives an optimal value of $\mu=2.7792$ for which $F_{2}=976.7225$. In the following simulations, to test the robustness of the controller, uncertainty of the form

$$
\xi(k)=\left[\begin{array}{lll}
0.3 & 0 & 0.3
\end{array}\right] x(k)
$$

has been included. Clearly $\|\xi\| \leq 0.3\|x\|$ and $0.3 \leq \sqrt{2.7792}$, from the theory developed earlier, asymptotic stability will be retained for a zero reference signal, and ultimate boundedness results will be achieved if $r_{s} \neq 0$.

Figures 1-2 show the response of the closed loop system obtained from implementing the above controller on the nominal and uncertain systems. Good output tracking is achieved in both situations, although of course, total invariance to the matched uncertainty is not obtained (Figure 1). In the nominal system there is no deviation from the sliding surface; however in the presence of uncertainty some deviation appears (Figure 2). Further results pertaining to the application of the theory developed in this paper to the HIRM aircraft benchmark are given in [15].

\section{Conclusions}

This paper has proposed a new output feedback based discrete-time sliding mode control scheme. It incorporates a tracking requirement and is dynamic in nature. Previous work has shown that with an appropriate choice of surface, discretetime sliding mode control can be applied to non-minimum phase systems. The original scheme was static output feedback in nature and so inherently this imposed restrictions on the class of systems to which it was applicable. The scheme which has been proposed here includes a compensator and so the output feedback restrictions have been removed. The key aspect of the new scheme proposed here is that it is still applicable to non-minimum phase systems.

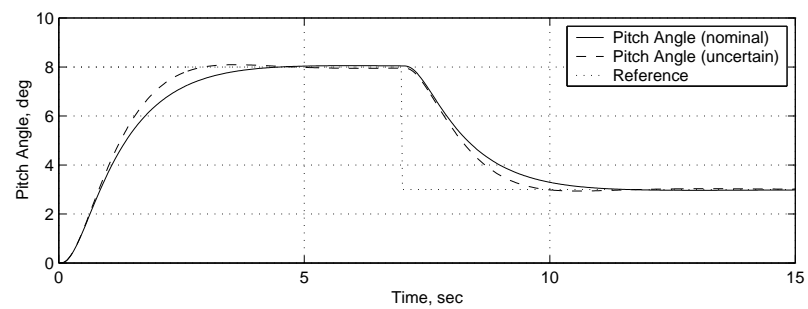

Fig. 1. Closed loop response to step changes in pitch angle

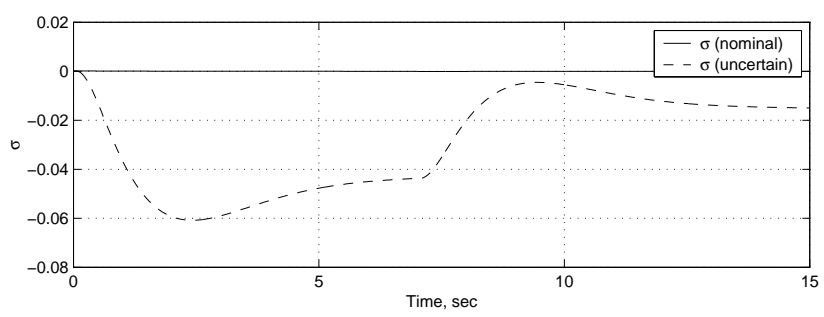

Fig. 2. Deviation from the sliding surface $\mathcal{S}$

\section{REFERENCES}

[1] S.H. Źak and S. Hui. On variable structure output feedback controllers for uncertain dynamical systems. IEEE Transaction on Automatic Control, 38(10):1509-1512, 1993.

[2] G. Bartolini, A. Pisano, and E. Usai. Digital second-order sliding mode control for uncertain nonlinear systems. Automatica, 37:1371-1377, 2001.

[3] S.P. Boyd, L. El-Ghaoui, E. Feron, and V. Balakrishnan. Linear Matrix Inequalities in Systems and Control Theory. Society for Industrial and Applied Mathematics, Philadelphia, 1994.

[4] C.Y. Chan. Robust discrete-time sliding mode controller. Systems and Control Letters, 23:371-374, 1994.

[5] C. Edwards and S. K. Spurgeon. Sliding mode stabilization of uncertain systems using only output information. International Journal of Control, 62:1129-1144, 1995.

[6] C. Edwards and S.K. Spurgeon. Robust output tracking using a sliding mode controller/observer scheme. International Journal of Control, 64:967-983, 1996.

[7] C. Edwards and S.K. Spurgeon. Sliding Mode Control: Theory and Applications. Taylor \& Francis, 1998.

[8] R. El-Khazali and R. DeCarlo. Output feedback variable structure control design. Automatica, 31(6):805-816, 1995.

[9] K. Furuta and Y. Pan. Variable structure control with sliding sector Automatica, 36:211-228, 2000.

[10] G. Golo and C. Milosavljević. Robust discrete-time chattering free sliding mode control. Systems and Control Letters, 41:19-28, 2000.

[11] S. Hui and S.H. Źak. On discrete-time variable structure sliding mode control. Systems and Control Letters, 38:283-288, 1999.

[12] A. Jafari Koshkouei and A. S. I. Zinober. Sliding mode control of discrete-time systems. Journal of Dynamic Systems, Measurement, and Control, 122:793-802, 2000.

[13] N.O. Lai, C. Edwards, and S.K. Spurgeon. On discrete time output feedback min-max controller. International Journal of Control, 77:554$561,2004$.

[14] N.O. Lai, C. Edwards, and S.K. Spurgeon. Discrete output feedback sliding mode control with integral action. International Journal of Robust and Nonlinear Control, 16:21-43, 2006.

[15] N.O. Lai, C. Edwards, and S.K. Spurgeon. An Implementation of an Output Tracking Dynamic Discrete-Time Sliding Mode Controller on an Aircraft Simulator. Proceedings of the IEEE Int. Workshop on VSS, Spain, 2006.

[16] J.F. Magni, S. Bennani, and J. Terlouw. Robust flight control: a design challenge. Springer-Verlag, 1997.

[17] G. Monsees. Discrete-Time Sliding Mode Control. PhD thesis, Delft University of Technology, 2002.

[18] N. Sharav-Schapiro, Z. J. Palmor, and A. Steinberg. Output stabilizing robust control for discrete uncertain systems. Automatica, 34:731-739, 1998.

[19] H. Sira-Ramirez. Non-linear discrete variable structure systems in quasisliding mode. International Journal of Control, 54:1171-1187, 1991.

[20] J. J. E. Slotine, J. K. Hedrick, and E. A. Misawa. On sliding observers for nonlinear systems. Transactions of the ASME: Journal of Dynamic Systems, Measurement and Control, 109:245-252, 1987.

[21] S.K. Spurgeon. Hyperplane design techniques for discrete-time variable structure control systems. International Journal of Control, 55:445-456, 1992.

[22] C.Y. Tang and E. Misawa. Sliding surface design for discrete VSS using LQR technique with a preset eigenvalue. Systems and Control Letters, 45:1-7, 2002.

[23] B. L. Walcott and S. H. Źak. Combined observer-controller synthesis for uncertain dynamical systems with applications. IEEE Transactions on Systems, Man and Cybernetics, 18:88-104, 1988. 